\title{
Multifield, Multifrequency Bosonic Stars and a Stabilization Mechanism
}

\author{
Nicolas Sanchis-Gual๑, ${ }^{1,2}$ Fabrizio Di Giovanni®${ }^{3}$ Carlos Herdeiro $\odot,{ }^{1}$ Eugen Radu, ${ }^{1}$ and José A. Font $\odot^{3,4}$ \\ ${ }^{1}$ Departamento de Matemática da Universidade de Aveiro and \\ Centre for Research and Development in Mathematics and Applications (CIDMA), \\ Campus de Santiago, 3810-183 Aveiro, Portugal \\ ${ }^{2}$ Centro de Astrofísica e Gravitação-CENTRA, Departamento de Física, Instituto Superior Técnico-IST, \\ Universidade de Lisboa-UL, Avenida Rovisco Pais 1, 1049-001 Lisboa, Portugal \\ ${ }^{3}$ Departamento de Astronomía y Astrofísica, Universitat de València, Dr. Moliner 50, 46100 Burjassot (València), Spain \\ ${ }^{4}$ Observatori Astronòmic, Universitat de València, C/ Catedrático José Beltrán 2, 46980 Paterna (València), Spain
}

(Received 29 March 2021; accepted 25 May 2021; published 17 June 2021)

\begin{abstract}
Scalar bosonic stars (BSs) stand out as a multipurpose model of exotic compact objects. We enlarge the landscape of such (asymptotically flat, stationary, everywhere regular) objects by considering multiple fields (possibly) with different frequencies. This allows for new morphologies and a stabilization mechanism for different sorts of unstable BSs. First, any odd number of complex fields, yields a continuous family of BSs departing from the spherical, equal frequency, $\ell$-BSs. As the simplest illustration, we construct the $\ell=1 \mathrm{BSs}$ family, that includes several single-frequency solutions, including even parity (such as spinning BSs and a toroidal, static BS) and odd parity (a dipole BS) limits. Second, these limiting solutions are dynamically unstable, but can be stabilized by a hybrid- $\ell$ construction: adding a sufficiently large fundamental $\ell=0 \mathrm{BS}$ of another field, with a different frequency. Evidence for this dynamical robustness is obtained by nonlinear numerical simulations of the corresponding Einstein-(complex, massive) Klein-Gordon system, both in formation and evolution scenarios, and a suggestive correlation between stability and energy distribution is observed. Similarities and differences with vector BSs are anticipated.
\end{abstract}

DOI: 10.1103/PhysRevLett.126.241105

Introduction.-Recent observations of dark compact objects, via gravitational waves [1-3], very large baseline interferometry imaging of M87* [4] or orbital motions near Sagittarius $A^{*}[5]$ support the black hole hypothesis. Yet, the issue of degeneracy remains a central question. This has been sharpened by recent illustrations, in both the gravitational and electromagnetic channels [6,7], using dynamically robust bosonic stars (BSs) to imitate the observed data.

In spite of many proposed black hole mimicker models [8], imposing an established formation mechanism and dynamical stability, within a sound effective field theory, restricts considerably the choices. The fundamental spherical (scalar $[9,10]$ or vector [11]) BSs, occurring in Einstein's gravity minimally coupled to a single complex, free bosonic field, fulfill these criteria [12], having become prolific testing grounds for strong-gravity phenomenology. The purpose of this Letter is to enlarge the landscape of dynamically robust BSs, by considering multifield, multifrequency solutions, which will open new avenues of research, both theoretical and phenomenological, for these remarkable gravitational solitons.

Single and multifield BSs._-Single field BSs appear in different varieties [13] besides the aforementioned fundamental spherical (monopole) solutions [14], including spinning BSs [11,15-17] and multipolar (static) BSs [18]. Concerning the former, only the vector case is dynamically robust [19]; concerning the latter, the simplest illustration is the dipole BS, shown to be unstable below.

Single-field BSs provide building blocks for multifield BSs, despite the nonlinearity of the model. Appropriate superpositions, moreover, change dynamical properties. An excited monopole scalar $\mathrm{BS}$, which is unstable against decaying to a fundamental BS [20], is stabilized by adding a sufficiently large fundamental monopole BS of a second field [21] (see also [22-24]). In the same spirit, for the nonrelativistic BSs of the Schrödinger-Poisson system, a dipole configuration is stabilized by adding a sufficiently large fundamental monopole [25] (see also [26]). These examples turn out to be illustrations of a stabilization mechanism, as we shall discuss.

A particular type of multifield BSs, composed of an odd number $\left(2 \ell+1, \ell \in \mathbb{N}_{0}\right)$ of (equal frequency) complex scalar fields was unveiled in [27] and dubbed $\ell$-BSs. These are spherical and can be seen as a superposition of all $m$ multipoles, with the same amplitude, for a given $\ell . \ell$-BSs were shown to be stable in spherical symmetry [28]; but nonspherical perturbations suggest new equilibrium configurations exist with different frequencies for different fields [29]. This will be confirmed herein: $\ell$-BS are just the 
symmetry-enhanced points of larger continuous families of multifield, multifrequency BSs [30].

The model.-Einstein's gravity minimally coupled to a set of $N$ free, complex, massive scalar fields $\Phi^{(j)}$ is $S=(16 \pi G)^{-1} \int d^{4} x \sqrt{-g}[R-\mathcal{L}]$, where $G$ is Newton's constant, $R$ the Ricci scalar and the matter Lagrangian is

$$
\mathcal{L}=\sum_{j=1}^{N}\left(g^{\alpha \beta} \Phi_{, \alpha}^{(j) *} \Phi_{, \beta}^{(j)}+\mu^{2} \Phi^{(j) *} \Phi^{(j)}\right)
$$

$\mu$ is the (common) mass of all fields $\Phi^{(j)}$ and the asterisk $(*)$ denotes complex conjugation.

All BSs studied herein are described by the metric ansatz $d s^{2}=-e^{2 F_{0}(r, \theta)} d t^{2}+e^{2 F_{1}(r, \theta)}\left(d r^{2}+r^{2} d \theta^{2}\right)+$ $e^{2 F_{2}(r, \theta)} r^{2} \sin ^{2} \theta(d \varphi-W(r, \theta) d t)^{2}$, in terms of four unknown metric functions of the coordinates $(r, \theta)$; the two Killing coordinates $(t, \varphi)$ represent the time and azimuthal directions. The $N$ scalar fields $\Phi^{(j)}$ are

$$
\Phi^{(j)}=\phi_{j}(r, \theta) e^{-i\left(w_{j} t-m_{j} \varphi\right)},
$$

where $w_{j} \in \mathbb{R}^{+}$are the fields' frequencies and $m_{j} \in \mathbb{Z}$ the azimuthal harmonic indices. The fields' amplitudes $\phi_{j}$ are real functions. This ansatz illustrates symmetry noninheritance [34]: each $\Phi^{(j)}$ depends on the Killing coordinates but its energy-momentum tensor (EMT) does not [35].

Constructing the enlarged $\ell$-BSs family.-Taking an odd number of fields, $N=2 \ell+1$, for a fixed $\ell \in \mathbb{N}_{0}$, a spherical ansatz $\left(W=0, F_{1}=F_{2}\right.$, with no angular dependence), equal frequencies $\left(w_{j}=w\right)$, and equal radial amplitudes such that $\phi_{j}(r, \theta) e^{i m_{j} \varphi}=f(r) Y_{\ell}^{-\ell-1+j}(\theta, \varphi)$, where $Y_{\ell}^{m}$ are the standard spherical harmonics, one obtains $\ell$-BSs [27].

Taking still $N=2 \ell+1$ but keeping the most general ansatz discussed above new possibilities emerge. We take $m_{j}=-\ell-1+j$, as for $\ell$-BSs. For concreteness we focus on the simplest nontrivial $\ell=1$ case. Then, the problem reduces to solving a set of seven partial differential equations (PDEs), for $F_{0,1,2}, W$, and $\phi_{1,2,3}$. This number reduces for particular cases [36]. These PDEs are solved with boundary conditions: (i) at $r=0$, $\partial_{r} F_{0,1,2}=0, \partial_{r} W=0, \partial_{r} \phi_{2}=\phi_{1,3}=0$; (ii) at infinity all functions vanish, $F_{0,1,2}=W=\phi_{i}=0$; (iii) at $\theta=0, \pi, \partial_{\theta} F_{0,1,2}=0, \partial_{\theta} \phi_{2}=\phi_{1,3}=0$; (iv) the geometry is invariant under a reflection along the equatorial plane $\theta=\pi / 2$, and, as for $\ell$-BSs, $\phi_{2}$ and $\phi_{1,3}$ are parity odd and even functions, respectively. Thus, at $\theta=\pi / 2$, $\partial_{\theta} F_{0,1,2}=\partial_{\theta} W=\phi_{2}=\partial_{\theta} \phi_{1,3}=0$. All configurations reported here are fundamental, with $n=0$, where $n$ is the number of nodes along the equatorial plane of $\phi_{1,3}(r, \pi / 2)$ [37]. The solutions are constructed numerically by employing the same approach as for the case of single-field BSs-see, e.g., the description in [38].

The single-frequency, multifield limits.-There are special limits where all fields have the same frequency $\left(w_{j}=w\right)$. First, there are two types of single-field configurations: (i) dipole $\mathrm{BSs}\left(\mathrm{DBS}_{0}\right)$, which are odd parity, obtained by taking only the $m=0$ mode, $\phi_{2} \neq 0$ [39] (see also [40]). Their angular momentum density vanishes $(W=0)$ and so does their total angular momentum, $J=0$; (ii) spinning $\mathrm{BSs}\left(\mathrm{SBS}_{ \pm 1}\right)$ [11,15-17], which are even parity and have $J \neq 0$, obtained by taking only either $\phi_{1} \neq 0\left(\mathrm{SBS}_{-1}\right)$ or $\phi_{3} \neq 0\left(\mathrm{SBS}_{+1}\right)$.

Second, combinations of single-field configurations lead to two types of two-field configurations: (iii) spinning dipolar BSs $\left(\mathrm{DBS}_{0}+\mathrm{SBS}_{ \pm 1}\right)$, in which case only either $\phi_{1} \neq \phi_{2} \neq 0 \quad\left(\mathrm{SBS}_{-1}+\mathrm{DBS}_{0}\right) \quad$ or $\quad \phi_{2} \neq \phi_{3} \neq 0$ $\left(\mathrm{DBS}_{0}+\mathrm{SBS}_{+1}\right)$. These are novel solutions with $J \neq 0$, carried by the even-parity scalar field; (iv) toroidal static BSs $\left(\mathrm{SBS}_{-1}+\mathrm{SBS}_{+1}\right)$, for which $\phi_{1}=\phi_{3} \equiv \phi \neq 0$. Each field $\Phi^{(1)}, \Phi^{(3)}$ carries a local angular momentum density, with the corresponding EMT component $T_{\varphi}^{t(1)}=-T_{\varphi}^{t(3)}=2 e^{-2 F_{0}} w \phi^{2}$, such that their sum is zero, $T_{\varphi}^{t}=0$, and the spacetime is locally and globally static, with $J=0$.

Finally, (v) $\ell$-BSs $\left(\mathrm{SBS}_{-1}+\mathrm{DBS}_{0}+\mathrm{SBS}_{+1}\right)$, which are static, spherical, and have $\phi_{i}=\phi(r)(\sin \theta / \sqrt{2}$, $\cos \theta, \sin \theta / \sqrt{2}$ ). Figure 1 illustrates the single-frequency limits of the enlarged $\ell=1 \mathrm{BSs}$ family as 3D plots.

For each of the five types of solutions described above, there is a one-dimensional family of BSs with $w_{\min }<w<\mu$, where $w_{\min }$ is family dependent. In an ADM mass, $M$ vs frequency $w$ diagram, they describe a spiral-type curve (costumary for BSs) -Fig. 2 [41]. As the frequency is decreased from the maximal value, $\mu$, the ADM mass increases up to a maximum value $M^{(\max )}$ which

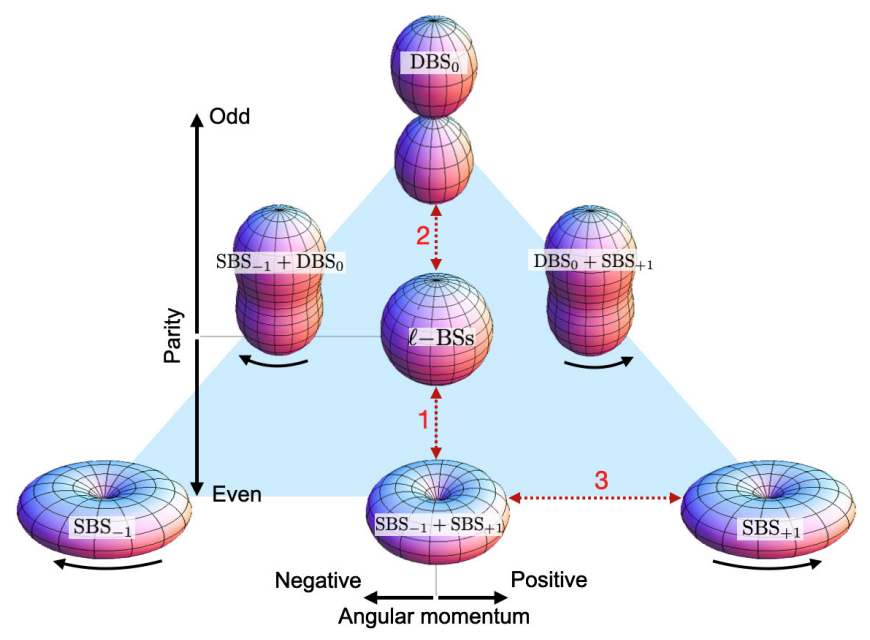

FIG. $1 . \quad \ell=1$ BSs family. 


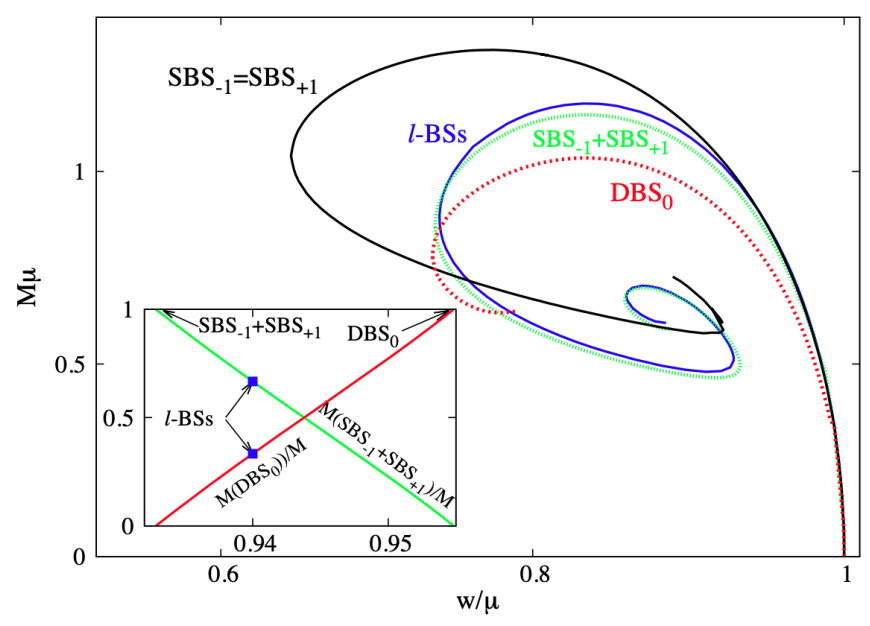

FIG. 2. ADM mass vs frequency for some of the singlefrequency limits of the $\ell=1 \mathrm{BSs}$ family. (Inset) Fraction of the total mass in $\mathrm{SBS}_{-1}+\mathrm{SBS}_{+1}$ and in $\mathrm{DBS}_{0}$ along the static sequence $1+2$ in Fig. 1 for $w_{2}=0.94 \mu$ (and $w_{1} / \mu=w_{3} / \mu$ in the $x$ axis).

is family dependent. The energy density distribution of the solutions is illustrated by the morphologies in Fig. 1.

The multifrequency, multifield interpolations.-Relaxing the equal frequency requirement a larger solution space emerges (blue triangle in Fig. 1). There are multifrequency BSs interpolating between the single-frequency ones, which are particular points in a manifold of solutions [42]. As an illustration consider the interpolation $\mathrm{SBS}_{-1}+\mathrm{SBS}_{+1} \leftrightarrow \mathrm{DBS}_{0}$, which goes through an $\ell$-BS. Fix, e.g., $w_{2}=0.94 \mu$ for the $\ell$-BS along the sequenceFig. 2 (inset). On the one hand, decreasing $w_{1}=w_{3}$ $\left(w_{2}\right.$ fixed), the toroidal static $\mathrm{BS}\left(\mathrm{SBS}_{-1}+\mathrm{SBS}_{+1}\right)$ is approached for $w_{1}=w_{3} \simeq 0.933 \mu$-sequence 1 in Fig. 1. On the other hand, increasing $w_{1}=w_{3}$ ( $w_{2}$ fixed), the dipole $\mathrm{DBS}_{0}$ is obtained for $w_{1}=w_{3} \simeq 0.955 \mu-$ sequence 2. These are static BSs sequences; thus $W=0$.

Similar interpolations occur between configurations with and without angular momentum, as in the transition $\mathrm{SBS}_{-1}+\mathrm{SBS}_{+1} \leftrightarrow \mathrm{SBS}_{+1}$ - sequence 3 in Fig. 1. Starting from a static, toroidal BS with $w_{1}=w_{3}=0.8 \mu$, varying $w_{3}$ ( $w_{1}$ fixed), the amplitude of $\Phi^{(1)}$ vanishes for a critical value of $w_{3} \simeq 0.829 \mu$, yielding the single-field $\mathrm{SBS}_{+1}$. All intermediate solutions with $w_{1} \neq w_{3}$ possess a nonvanishing angular momentum. In all sequences, a similar picture holds considering other frequencies.

The manifold of solutions of the $\ell=1 \mathrm{BSs}$ family is as follows. Starting from an $\ell$-BS with a fixed frequency $w_{1}=w_{2}=w_{3}$, the line of static $(J=0) \mathrm{BSs}$ is obtained keeping $w_{1}=w_{3}$ and varying the ratio $y \equiv w_{1} / w_{2}$ $\left(=w_{3} / w_{2}\right)$. Then $y \in\left[y_{\min }, y_{\max }\right] . y$ varies the parity of the BSs; the boundary values are the parity even and odd solutions, respectively. Then, for each fixed $y$ one can vary $x \equiv w_{3} / w_{1}$, with $x \in\left[x_{\min }, x_{\max }\right]$, where the limits are $y$ dependent. $x$ varies $J$; for $x>1(x<1), J$ is positive

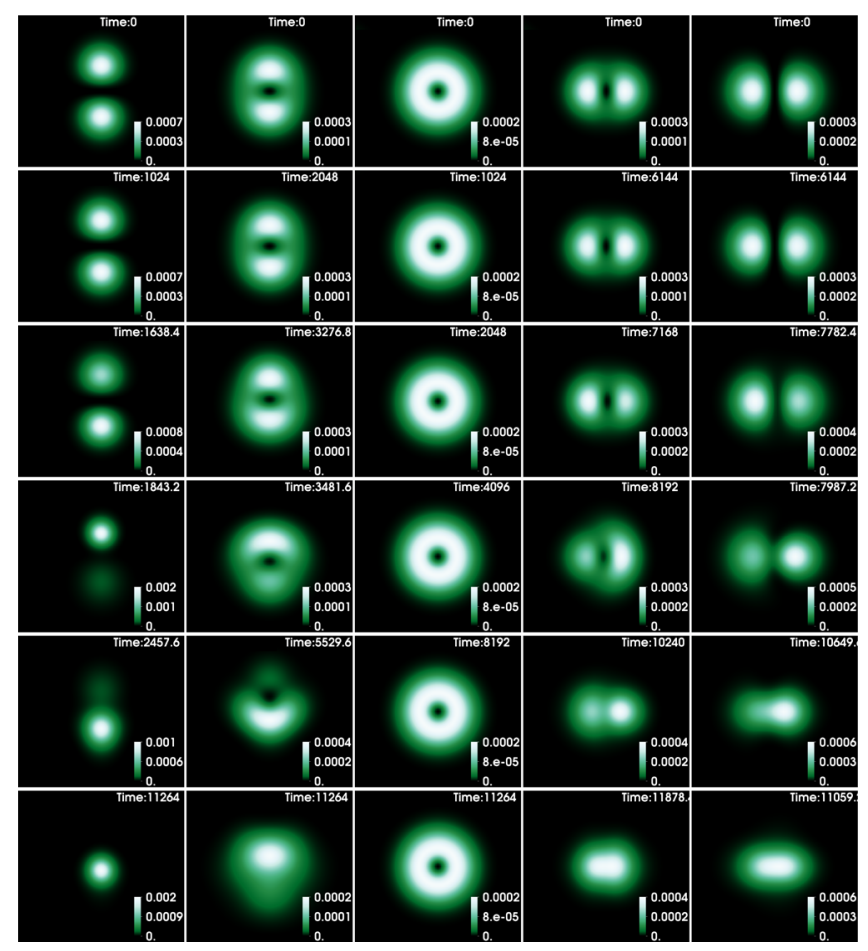

FIG. 3. Time evolutions of the energy density for five static BSs in the $\ell=1$ family. Each column shows six sequential snapshots of the $x z(y=0)$ plane (time running from top to bottom). From left to right the values of $w_{1}=w_{3}$ of the five models are: 0.955 $\left(\mathrm{DBS}_{0}\right), 0.945,0.940(\ell-\mathrm{BS}), 0.935,0.933\left(\mathrm{SBS}_{-1}+\mathrm{SBS}_{+1}\right)$.

(negative) [43]. Finally, varying the frequency of the starting $\ell$-BS yields a 3D manifold of solutions. Thus, we expect a $(2 \ell+1) D$ manifold of multifrequency, multifield BSs for a model with $(2 \ell+1)$ complex scalar fields, including $\ell$-BSs as symmetry-enhanced solutions.

Dynamical (in)stability.-We assess the dynamical stability of representative solutions in the $\ell=1 \mathrm{BS}$ family by resorting to fully nonlinear dynamical evolutions of the corresponding Einstein-(multi-)Klein-Gordon system. The infrastructure used in the numerical evolutions is the same as in [19].

Figure 3 exhibits the results for a sequence of static solutions (i.e., along sequences 1 and 2 in Fig. 1, including the dipole, the $\ell$-BS and the toroidal static BS). We find that all solutions (except the $\ell$-BS) are dynamically unstable, decaying to a multifield BS in which all fields have $\ell=m=0$. Including $J$ does not improve dynamical stability. The $\mathrm{SBS}_{ \pm 1}$ are unstable against a nonaxisymmetric instability [19] and all hybrid cases we have studied (such as $\mathrm{SBS}_{ \pm 1}+\mathrm{DBS}_{0}$ ) also decay to the fundamental $\ell=0$ BSs.

Hybrid $\ell$-BSs and a stabilization mechanism.-Instead of focusing on a single $\ell$-BSs family we now allow superpositions of such families with different $\ell$ 's. The most elementary example is to add $\ell=0 \mathrm{BSs}$ to the $\ell=1$ family. Thus we add a fourth complex scalar field $\Phi^{(0)}$, 
obeying (1) and (2) with $j=0$ (hence, now $j=0,1,2,3$ ) and $m_{j}=0$. Its boundary conditions are $\left.\partial_{r} \phi_{0}\right|_{r=0}=$ $\left.\phi_{0}\right|_{r=\infty}=\left.\partial_{\theta} \phi_{0}\right|_{\theta=0, \pi}=0$, besides being parity even. Keeping only $\Phi^{(0)}$, the basic solution is the single-field, fundamental, monopole BS $\left(\mathrm{MBS}_{0}\right)$. We now show that adding $\mathrm{MBS}_{0}$ can quench the instabilities observed in the $\ell=1$ family.

To be concrete we consider the following superpositions: (A) $\mathrm{MBS}_{0}+\mathrm{SBS}_{+1}$ and (B) $\mathrm{MBS}_{0}+\mathrm{DBS}_{0}$. As an illustration of (A), fixing $w_{3} / \mu=0.98$, there is a continuous sequence of solutions reducing to the $\mathrm{MBS}_{0}\left(\mathrm{SBS}_{+1}\right)$ for $w_{0} / \mu=0.964$ (0.975). We refer to the intermediate configurations as "saturns." Their dynamical evolutionsFig. 4-exhibit a simple pattern: sufficiently close to the $\mathrm{MBS}_{0}\left(\mathrm{SBS}_{+1}\right)$ limit, saturns are stable (unstable). Here, stability means no sign of instabilities for long evolutions $(t \simeq 24000)$ [44]. Attempting to interpret the transition between the two regimes, we observe a correlation between instability and the $r$ coordinate of the maximum of the

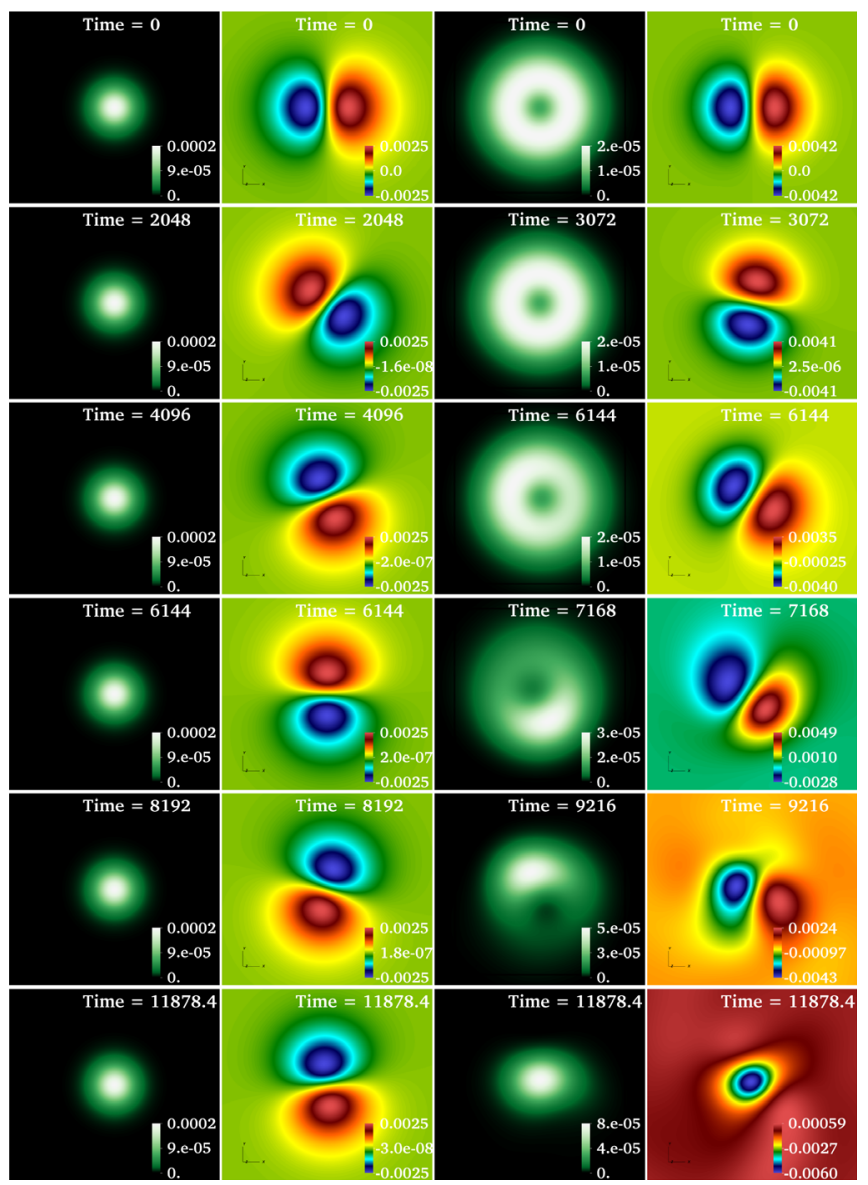

FIG. 4. Time evolution (top to bottom) of two saturns: (left and middle left) $\omega_{1} / \mu=0.967$; (middle right and right) $\omega_{1} / \mu=0.974$. For each case the energy density and the real part of $\Phi^{(3)}$ on the $x y$ plane are shown. The first (second) saturn is close to $\mathrm{MBS}_{0}\left(\mathrm{SBS}_{+1}\right)$ and is stable (unstable). energy density-Fig. 5: when the latter is at the origin $(r=0)$ no instability is observed.

As an example of (B), fixing $w_{0} / \mu=0.97$, there is a continuous sequence of solutions reducing to the $\mathrm{MBS}_{0}$ $\left(\mathrm{DBS}_{0}\right)$ for $w_{2} / \mu=0.983$ (0.973). We refer to the intermediate configurations as "pods." Evolving this sequence of pods reveals analogous patterns: (a) sufficiently close to the $\mathrm{MBS}_{0}\left(\mathrm{DBS}_{0}\right)$ limit, pods are stable (unstable)—see Sec. I of Supplemental Material [45] for snapshots of the evolutions; (b) when the energy density maximum, which, in general, has two symmetric points located on the $z$ axis (i.e., $\theta=0, \pi)$ is at the origin, no instability is observedFig. 5 (inset).

Generality and remarks.-An analogous family of vector $\ell=1$ BSs should exist. Preliminary results show an important difference: the whole sequence 3 (see Fig. 1) is stable in the vector case, including the $\mathrm{SBS}_{-1}+\mathrm{SBS}_{+1}$ static configuration, which is now spheroidal rather than toroidal (see Sec. II of Supplemental Material [45] for details). This is a consequence of the stability of vector $\mathrm{SBS}_{ \pm 1}$ [19]. On the other hand, we have evidence that the vector $\mathrm{DBS}_{0}$ is unstable, as in the scalar case (see Sec. III of Supplemental Material [45]).

A byproduct of our construction is the realization that all single-frequency BSs arising in (combinations of) models of type (1) are continuously connected within a multidimensional solution manifold, interpolated by multifrequency solutions. For instance, spherical $\left(\mathrm{MBS}_{0}\right)$ and spinning $\left(\mathrm{SBS}_{ \pm 1}\right) \mathrm{BSs}$, typically described as disconnected, are connected (via saturns).

Adding the fundamental $\mathrm{MBS}_{0}$, which is the ground state of the whole family, stabilizes different types of

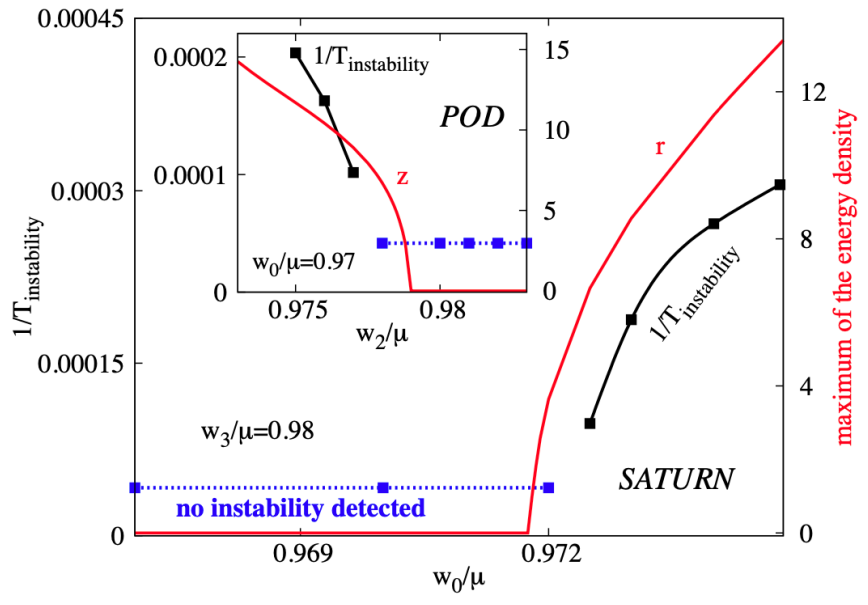

FIG. 5. Instability timescale (black dots and lines) and maximum of the total energy density (red lines) for the sequence of saturns (main panel) and pods (inset) described in the text. The blue points and line are thresholds when no instability is seen. The $z$ coordinate in the inset is $r$ for $\theta=0, \pi$. $T_{\text {instability }}$ refers to the time when we observe that the solution begins to clearly deviate from axisymmetry for saturns and from equatorial symmetry for pods. 
unstable BSs, such as excited monopole BSs [21], spinning BSs, and dipole BSs. Such stable configurations can actually form from the (incomplete) gravitational collapse of dilute distributions of the corresponding fields and multipoles-see Sec. III of Supplemental Material [45]. It would be interesting to probe the generality of this cooperative stabilization mechanism, and if other (higher $\ell$, say) multipolar BSs can be stabilized similarly [50].

Another mechanism for mitigating instabilities is adding self-interactions [52], which was suggested to quench the instability of spinning BSs, without requiring the energy density to be maximized at the origin [53]. It would be interesting to construct the corresponding $\ell=1$ BSs family in models with self-interactions and investigate whether other members of the family can be stabilized by self-interactions.

Finally, among the possible generalizations, one could consider different masses for the fields in (1). At least for slightly different masses multifield, multifrequency solutions still exist, albeit with a modified solution space, for instance without an exactly spherically symmetric composite state. Different generalizations could be obtained by introducing a target space metric (see, e.g., $[54,55])$ in the multifield model.

We thank Darío Núñez, Víctor Jaramillo, Argelia Bernal, Juan Carlos Degollado, Juan Barranco, Francisco Guzmán and Luis Ureña-López, for useful discussions and valuable comments. This work was supported by the Spanish Agencia Estatal de Investigación (Grant No. PGC2018095984-B-I00), by the Generalitat Valenciana (PROMETEO/2019/071 and GRISOLIAP/2019/029), by the Center for Research and Development in Mathematics and Applications (CIDMA) through the Portuguese Foundation for Science and Technology (FCTFundação para a Ciência e a Tecnologia), references UIDB/04106/2020 and UIDP/04106/2020, by national funds (OE), through FCT, I.P., in the scope of the framework contract foreseen in the numbers 4,5 , and 6 of article 23, of the Decree-Law 57/2016, changed by Law 57/2017 and by the projects PTDC/FIS-OUT/28407/2017, CERN/FIS-PAR/0027/2019 and PTDC/FIS-AST/3041/ 2020. This work has further been supported by the European Union's Horizon 2020 research and innovation (RISE) programme H2020-MSCA-RISE-2017 Grant No. FunFiCO-777740 and by FCT through Project No. UIDB/00099/2020. We would like to acknowledge networking support by the COST Action GWverse CA16104. Computations have been performed at the Servei d'Informàtica de la Universitat de València, the Argus and Blafis cluster at the U. Aveiro and on the "Baltasar Sete-Sois" cluster at IST.

[1] B. P. Abbott, R. Abbott, T. D. Abbott, M. R. Abernathy, F. Acernese, K. Ackley, C. Adams, T. Adams, P. Addesso,
R. X. Adhikari et al. (LIGO Scientific and Virgo Collaborations), Phys. Rev. Lett. 116, 061102 (2016).

[2] B. P. Abbott et al. (LIGO Scientific and Virgo Collaborations), Phys. Rev. X 9, 031040 (2019).

[3] R. Abbott et al. (LIGO Scientific and Virgo Collaborations), arXiv:2010.14527 [Phys. Rev. X (to be published)].

[4] K. Akiyama et al. (Event Horizon Telescope Collaboration), Astrophys. J. 875, L1 (2019).

[5] R. Abuter, A. Amorim, M. Bauböck, J. P. Berger, H. Bonnet, W. Brandner, Y. Clénet, V. Coudé Du Foresto, and P. T. de Zeeuw (Gravity Collaboration), Astron. Astrophys. 618, L10 (2018).

[6] J. Calderón Bustillo, N. Sanchis-Gual, A. Torres-Forné, J. A. Font, A. Vajpeyi, R. Smith, C. Herdeiro, E. Radu, and S. H. W. Leong, Phys. Rev. Lett. 126, 081101 (2021).

[7] C. A. R. Herdeiro, A. M. Pombo, E. Radu, P. V. P. Cunha, and N. Sanchis-Gual, J. Cosmol. Astropart. Phys. 04 (2021) 051.

[8] V. Cardoso and P. Pani, Living Rev. Relativity 22, 4 (2019).

[9] D. J. Kaup, Phys. Rev. 172, 1331 (1968).

[10] R. Ruffini and S. Bonazzola, Phys. Rev. 187, 1767 (1969).

[11] R. Brito, V. Cardoso, C. A. R. Herdeiro, and E. Radu, Phys. Lett. B 752, 291 (2016).

[12] S. L. Liebling and C. Palenzuela, Living Rev. Relativity 15, 6 (2012).

[13] In this Letter we focus on non-self-interacting fields.

[14] C. A. R. Herdeiro, A. M. Pombo, and E. Radu, Phys. Lett. B 773, 654 (2017).

[15] F. E. Schunck and E. W. Mielke, Phys. Lett. A 249, 389 (1998).

[16] S. Yoshida and Y. Eriguchi, Phys. Rev. D 56, 762 (1997).

[17] C. Herdeiro, I. Perapechka, E. Radu, and Y. Shnir, Phys. Lett. B 797, 134845 (2019).

[18] C. A. R. Herdeiro, J. Kunz, I. Perapechka, E. Radu, and Y. Shnir, Phys. Lett. B 812, 136027 (2021).

[19] N. Sanchis-Gual, F. Di Giovanni, M. Zilhão, C. Herdeiro, P. Cerdá-Durán, J. A. Font, and E. Radu, Phys. Rev. Lett. 123, 221101 (2019).

[20] J. Balakrishna, E. Seidel, and W.-M. Suen, Phys. Rev. D 58, 104004 (1998).

[21] A. Bernal, J. Barranco, D. Alic, and C. Palenzuela, Phys. Rev. D 81, 044031 (2010).

[22] T. Matos and L. A. Urena-Lopez, Gen. Relativ. Gravit. 39, 1279 (2007).

[23] L. A. Urena-Lopez and A. Bernal, Phys. Rev. D 82, 123535 (2010).

[24] F. Di Giovanni, S. Fakhry, N. Sanchis-Gual, J. C. Degollado, and J. A. Font, Phys. Rev. D 102, 084063 (2020).

[25] F. S. Guzmán and L. A. Ureña López, Phys. Rev. D 101, 081302(R) (2020).

[26] F. S. Guzmán, Astron. Nachr. 342, 398 (2021).

[27] M. Alcubierre, J. Barranco, A. Bernal, J. C. Degollado, A. Diez-Tejedor, M. Megevand, D. Nunez, and O. Sarbach, Classical Quantum Gravity 35, 19LT01 (2018).

[28] M. Alcubierre, J. Barranco, A. Bernal, J. C. Degollado, A. Diez-Tejedor, M. Megevand, D. Núñez, and O. Sarbach, Classical Quantum Gravity 36, 215013 (2019).

[29] V. Jaramillo, N. Sanchis-Gual, J. Barranco, A. Bernal, J. C. Degollado, C. Herdeiro, and D. Núñez, Phys. Rev. D 101, 124020 (2020). 
[30] In spherical symmetry, multifrequency BSs were discussed in [31]; and BSs in multiscalar theories were recently constructed in $[32,33]$.

[31] M. Choptuik, R. Masachs, and B. Way, Phys. Rev. Lett. 123, 131101 (2019).

[32] S. S. Yazadjiev and D. D. Doneva, Phys. Rev. D 99, 084011 (2019).

[33] L. G. Collodel, D. D. Doneva, and S. S. Yazadjiev, Phys. Rev. D 101, 044021 (2020).

[34] I. Smolić, Classical Quantum Gravity 32, 145010 (2015).

[35] For the $\ell$-BS, moreover, the fields depend on both the Killing and $\theta$ coordinates but the total EMT does not, albeit the individual EMT of each scalar field may still depend on the $\theta$ coordinate.

[36] The nonvanishing Einstein equations are $E_{t}^{t}, E_{r}^{r}, E_{\theta}^{\theta}, E_{\varphi}^{\varphi}, E_{\varphi}^{t}$, and $E_{r}^{\theta}$. Four of them are solved together with the three Klein-Gordon equations, yielding a coupled system of seven PDEs on the unknown functions $F_{0,1,2}, W, \phi_{1,2,3}$. The remaining two Einstein equations are treated as constraints and used to check the numerical accuracy.

[37] Excited solutions with $n>0$ exist as well.

[38] C. Herdeiro and E. Radu, Classical Quantum Gravity 32, 144001 (2015).

[39] It is $\Phi_{(2,1,0)}$ in the notation of [18].

[40] C. A. R. Herdeiro, J. Kunz, I. Perapechka, E. Radu, and Y. Shnir, Phys. Rev. D 103, 065009 (2021).

[41] The same holds for the Noether charge of the fields.

[42] Bear in mind each of these points is in fact a continuous family, spanning a range of frequencies.

[43] Since $J$ is carried by even parity fields, the $x$ range is maximal (minimal) for $y_{\min }\left(y_{\max }\right)$. This explains the triangular shape in Fig. 1.
[44] The typical size of the BSs here is around $r \sim 15-30$; then $t \simeq 24000$ means 400-800 light-crossing times.

[45] See Supplemental Material at http://link.aps.org/ supplemental/10.1103/PhysRevLett.126.241105 further details on the numerical evolution code and the construction of constraint-satisfying initial data for the formation scenario. We also show numerical results of the stability of pod configurations and the dynamical formation of multi-field bosonic stars, including a couple of examples of vector boson stars, which includes [19,46-49]

[46] H. Witek, M. Zilhao, G. Ficarra, and M. Elley, Canuda: A public numerical relativity library to probe fundamental physics (2020), https://dx.doi.org/10.5281/zenodo.3565475.

[47] M. Zilhão, H. Witek, and V. Cardoso, Classical Quantum Gravity 32, 234003 (2015).

[48] E. Seidel and W.-M. Suen, Phys. Rev. Lett. 72, 2516 (1994).

[49] F. Di Giovanni, N. Sanchis-Gual, C. A. R. Herdeiro, and J. A. Font, Phys. Rev. D 98, 064044 (2018).

[50] See also [51] for another multistate construction.

[51] Y.-B. Zeng, H.-B. Li, S.-X. Sun, S.-Y. Cui, and Y.-Q. Wang, arXiv:2103.10717.

[52] F. Di Giovanni, N. Sanchis-Gual, P. Cerdá-Durán, M. Zilhão, C. Herdeiro, J. A. Font, and E. Radu, Phys. Rev. D 102, 124009 (2020).

[53] N. Siemonsen and W. E. East, Phys. Rev. D 103, 044022 (2021).

[54] D. D. Doneva and S. S. Yazadjiev, Phys. Rev. D 101, 064072 (2020).

[55] V. I. Danchev, D. D. Doneva, and S. S. Yazadjiev, Eur. Phys. J. C 80, 878 (2020). 